\title{
Optimization of Laser-Ablation Micromachining by Choice of Scanning Algorithms and Use of Laser-Induced- Breakdown Spectroscopy
}

\author{
A. Baskevicius, A. Alesenkov, G. Chozevskis, J. Litvaityte, O. Balachninaite, D. Paipulas, A. Melninkaitis, V. Sirutkaitis
}

\author{
Laser Research Center, Vilnius University, Sauletekio ave. 10, LT-10223 Vilnius, Lithuania \\ E-mail:ona.balachninaite@ff.vu.lt
}

\begin{abstract}
Femtosecond-laser ablation for the microfabrication of 30- to 300- $\mu \mathrm{m}$-wide grooves with a depth to width ratio up to 2 in stainless steel was investigated. A laser based on $\mathrm{Yb}: \mathrm{KGW}$ with 300-fs pulse duration, $350-\mathrm{kHz}$ repetition rate and $6-\mathrm{W}$ average power was used. The influence of micromachining strategies, scanning methods and beam shapes on the precision and the speed of material removal were studied. Laser-induced-breakdown spectroscopy was used to monitor the micromachining process. The choice of the scanning pattern was found important to form rectangular grooves with high precision in high fluency ablation conditions.
\end{abstract}

DOI:10.2961/jlmn.2013.01.0006

Keywords: femtosecond laser micromachining, ultrafast laser induced breakdown spectroscopy, micromachining process monitoring

\section{Introduction}

Material processing such as laser drilling and cutting is one of the promising potential applications of femtosecond lasers in an industrial context. Femtosecond-laser material microprocessing based on ablation has a universal character. Nearly arbitrary micrometer-size patterns and structures can be fabricated [1-5]. The main influencing parameters for femtosecond laser ablation are: a) pulse fluence, b) pulse duration, c) pulse repetition rate, e) focused spot size and f) hatching scheme.

Two fluence regimes have been observed for the metals ablation for femtosecond (and shorter than $10 \mathrm{ps}$ ) pulses: first at low fluences up to $1-1.5 \mathrm{~J} \mathrm{~cm}^{-2}$ and second at high fluences up to $20 \mathrm{~J} \mathrm{~cm}^{-2}$ [6-7]. While in the low fluence regime the penetration depth is close to the theoretical optical penetration depth and ablation thresholds are low, at higher fluences the effective heat penetration depth is 10 20 times greater with accompanying higher ablation thresholds. Even in the femtosecond range, thermal ablation processes occur and reduce the quality and the accuracy of micromachining but increase the ablation efficiency. A systematic study of the influence of laser pulse width ranging from $110 \mathrm{fs}$ to $10 \mathrm{ps}$ for laser micromachining of stainless steel [8] showed that melting damage can be avoided for pulses shorter than 2.5 ps. Including other influencing factors, pulse durations shorter than 1 ps are optimal for micromachining of steel.

In micromachining of grooves it was shown, that the processing time reduction is proportional to the increase of the repetition rate from 1 to $15 \mathrm{kHz}$ [9]. Two distinctive effects influencing the drilling efficiency at high repetition rates: particle shielding and heat accumulation allow efficient usage of lasers with repetion rate in range of 100$600 \mathrm{kHz}$ depending on pulse energy and material properties [10]. Those results open the way to shorter manufacturing times with higher repetition rates preserving the same quality. This is a huge improvement for femtosecond machining in industrial applications, which require faster processing time to be competitive.

It becomes prohibitively difficult to scale the average power of femtosecond Ti:Sapphire lasers much beyond $10 \mathrm{~W}$ and amplified-pulse repetion rate beyond $10 \mathrm{kHz}$ [11]. Therefore the need for alternative femtosecond laser sources complementing the existing ones for microprocessing is obvious. Ytterbium $\left(\mathrm{Yb}^{3+}\right)$ doped gain media are one of promising laser materials for the next generation of diode-pumped high-power lasers [12, 13]. Femtosecond lasers based on ytterbium doped materials allows generation of 200-1000 fs pulses in bulk, thin disk and fiber configurations with average power from 1 to 200 $\mathrm{W}$ and pulse repetition rate in the range $1-1000 \mathrm{kHz}$ [1417]. Lasers based on these materials hold great promise for femtosecond micromachining as they are capable to increase ablation rate 5-100 times.

Other important parameters for the formation of micrometer size grooves are the cutting velocity, the number of cutting repetitions, and the focusing technique. Previously micromachining of grooves was performed mainly with 1-kHz Ti:Sapphire laser systems [7, 18-19], although advantages of multi-kilohertz femtosecond systems for machining has been affirmed [20-22].

The main goal of the work presented here was to examine the use of a high-repetition rate (up to $350 \mathrm{kHz}$ ) femtosecond laser system based on $\mathrm{Yb}: \mathrm{KGW}$ in the micromachining of the rectangular grooves in steel. Grove 
widths in the range of 30-200 $\mu \mathrm{m}$ and depth-to-width ratios up to 2 were investigated. Scanning geometries and the number of repetitions of the scanning path were considered as variables for achieving high precision and high material removal speed. Influence of the micromachining strategies, scanning methods and beam shapes on the precision and material removal speed was investigated. In order to monitor the micromachining process laser-induced-breakdown spectroscopy measurements were performed.

\section{Experimental set-up}

The optical-mechanical system used for machining grooves consisted of the laser, optical beam delivery components, a 3D linear stage positioning system, computer and electronic control units. In order to withstand high intensity, dielectric mirrors were selected for delivery of the femtosecond laser beam to the sample. Only three refractive components (a crystal-quartz half-wave plate, a thin-film polarizer on a fused silica substrate and a fused silica lens) were used in the system. The wave plate mounted on motorized rotation stage and in combination with the reflective thin-film polarizer was used as a variable attenuator. The method of "moving sample" while keeping laser's beam focus at fixed position was implemented with submicron position resolution. The positioning system consisted of three linear translation stages (Aerotech), which allow fast and accurate sample positioning. The accuracy of XY stages was $\sim 0.3$ $\mu \mathrm{m}$, and the maximum travel speed was $30 \mathrm{~cm} / \mathrm{s}$. This gave plausibility to having a short processing time with a highaverage-power laser. Also intellSCAN10 galvanometric scanners (from SCANLab $\mathrm{GmbH}$ ) were used for testing micromachining using the "moving beam" approach. The femtosecond laser (PHAROS, Light Conversion, Ltd.) used in the experiments was based on $\mathrm{Yb}: \mathrm{KGW}$, had pulse duration $\sim 300$ fs at 1030 -nm wavelength, repetition rate up to $350 \mathrm{kHz}$ and $6-\mathrm{W}$ average power. The operation of the PHAROS system was controlled by a set of microcontrollers that were linked together by an industrial CAN bus. In order to control hardware devices such as a laser and linear stage systems, SCA software (Altechna Ltd.) was used. The program supported the hardware and the creation of different object moving trajectories for special tasks. The focused beam diameter was changed by changing focusing lens. All grooves were characterized with an optical profilometer (Sensofar PL $\mu$ 2300). The bottom of the groove was characterized by SEM microscope (Hitachi TM-1000). Samples used for the experiment were non-polished plates of stainless steel with thickness of $3 \mathrm{~mm}$. Prior to laser machining all samples were cleaned in acetone and after the fabrication washed in an ultrasonic bath filled with isopropanol for 10 minutes.

\section{Results}

\subsection{Influence of the micromachining strategies on the groove structure}

Several different micromachining strategies could be applied for precise cutting of steep-walled grooves on a steel surface. The two most common laser processing techniques are linear sample translation in respect to fixed focus posi- tion and the scanning beam approach where laser beam is moved in respect to fixed sample position by means of galvanometric scanning and focused using an f-theta lens. Each technique has its own advantages and disadvantages and should be chosen according to the application. A system of linear translation stages in combination with a high numerical aperture (NA) objective can be very precise in beam positioning, and the maximum traveling range (processing area) of such stages could be quite high. However, sample translation speed tends to be limited. The beam scanning approach does not have such drawback, but on other hand, it has limited processing area which is linked to the choice of focusing lens. With beam scanning large processing areas require low NA value lenses, and this can be an issue with power-limited laser sources in achieving radiation intensities required for efficient ablation. Both these techniques were tested for groove micromachining tasks.

\subsubsection{Microfabrication using linear stages}

The steel sample was attached to the linear translating stage, and laser beam was focused on the surface with $25-\mathrm{mm}$ focusing lens (nominal NA value of 0.1) to spot diameter of $10 \mu \mathrm{m}$. Since this diameter was smaller than the required groove width, additional width was obtained with the implemented patterning algorithm.

Two conventional scanning patterns were examined for groove fabrication: the raster algorithm (Fig. 1) and the spiral algorithm (Fig. 2). With raster patterning, the required groove width was achieved by continuously translating sample up and down the groove and constantly shifting new line position by constant $d$. In such patterning grooves are always cut straight from one edge to the other. In the spiral patterning algorithm, the sample is translated in a spiral path with respect to the beam focus having pitch length $d$. In this configuration, groove cutting progresses from the groove center towards the walls.

The laser pulse energy was $20 \mu \mathrm{J}(2 \mathrm{~W}$ at $100 \mathrm{kHz})$, and the energy fluence in the focus was as large as $30 \mathrm{~J} / \mathrm{cm}^{2}$. This value corresponds to strong ablation regime [22] that leads to the efficient generation of ablation debris.

The groove produced with raster patterning algorithm is unsymmetrical because majority of the ablated particles tend to stick to one side of the grove (Fig. 1b). This can be understood by comparing the ablation conditions at the first raster lines with respect to last: the last ablated line tend to pollute the previous ones, leading to asymmetric walls. To get deeper grooves, several repetitions of the patterning algorithm are necessary, and such debris accumulation becomes more evident. The spiral patterning shows much better results in terms of groove rectangularity and wall verticality (Fig. 2b). However, at the center of the bottom surface of the fabricated groove, a hill formed from adhered microparticles was observed. Those formations are particularly hard to remove; the accumulated particles remained even after cleaning the sample in the ultrasonic bath. 
It was possible to reduce the debris sticking to the groove bottom by applying an inert gas (argon) jet to the ablating region. The gas jet tended to blow away debris from the groove improving the rectangularity. However, as seen from the Fig. 3, the jet presence reduced ablation efficiency by almost at half. The groove bottom surface become flat-

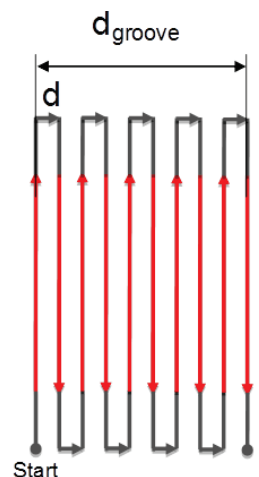

a)

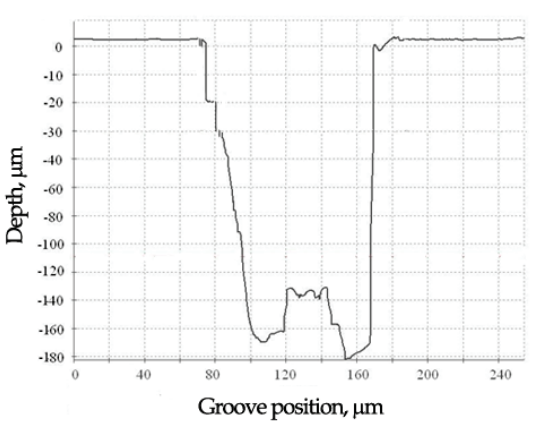

b)
Fig. 1. Raster patterning algorithm (a) and groove profile after patterning with hatch algorithm (b). The displacement size $(d)$ is $5 \mu \mathrm{m}$. The unsymmetrical slopes show the drawback of this algorithm.

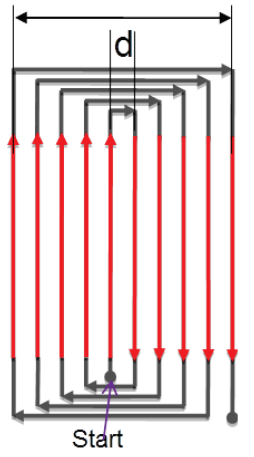

a)

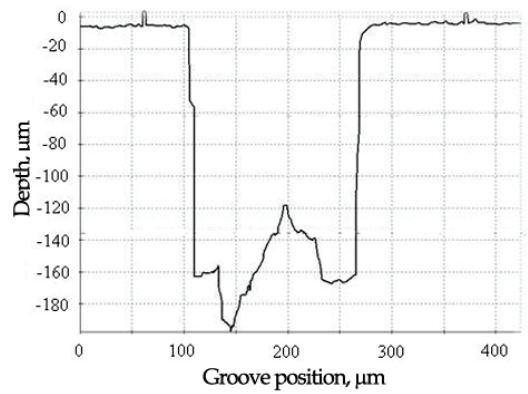

b)
Fig. 2. Spiral algorithm (a) and groove profile after spiral algorithm patterning (b). The pitch size $(d)$ is $5 \mu \mathrm{m}$. Slopes are symmetric and steep, debris are accumulated only at the bottom of the groove.

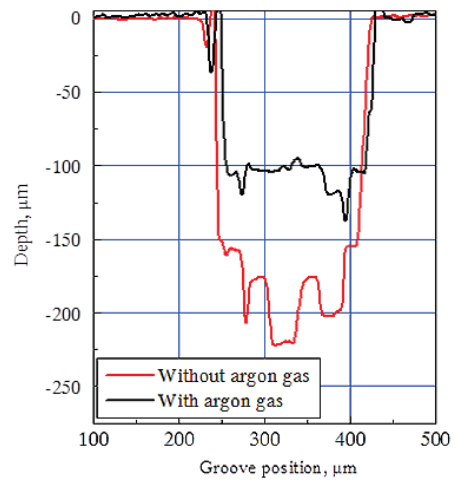

a)

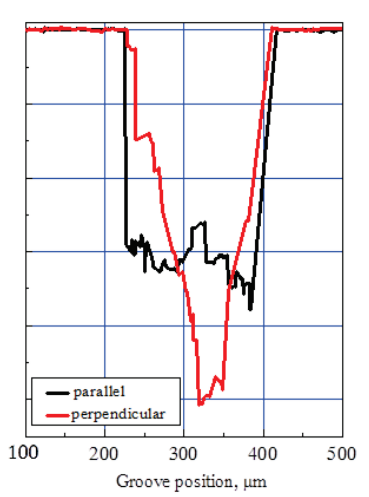

b)
Fig. 3.Groove profiles made in steel a) with and without argon gas injection; b) with different polarization in respect to groove orientation. Processing parameters: $2 \mathrm{~W}$ at 100 $\mathrm{kHz}$. Sample translation speed in a) $40 \mathrm{~mm} / \mathrm{s}$; b) $30 \mathrm{~mm} / \mathrm{s}$. Spiral patterning algorithm with 10 repetitions and pitch size $-5 \mu \mathrm{m}$. ter, but shallower. This can be explained, by the fact that hot microparticles generated during ablation increase laser irradiation absorption and slow the ablation process.

Laser polarization also strongly influences rectangularity of the fabricated groove. In order to reduce reflections from the wall it was essential to use laser polarization that is parallel to the groove. With polarization perpendicular to the wall, V-shaped grooves were formed (Fig 3b).

The experiments that determined the optimal displacement of adjacent scan lines $(d)$ were also carried out. It was found out that at least $50 \%$ of ablated regions should overlap in order to get smooth-bottom structures.

The width of the groove is determined by the number of scanned lines in the algorithm, while the depth is scaled by repetitive scanning. As example, to fabricate a $280-\mu \mathrm{m}$ wide and $170-\mu \mathrm{m}$ deep groove, the required algorithm had 50 raster lines (separation between lines of $5 \mu \mathrm{m}$ ) and 28 repetitions, using $20 \mu \mathrm{J}$ pulse energies at $100 \mathrm{kHz}$. Sample translation speed was $100 \mathrm{~mm} / \mathrm{s}$. Total cutting time for a 1$\mathrm{cm}$ length groove was 15 minutes. The resulting groove is shown in Fig 4. No gas injection was used in this particular experiment. To form flat bottom we used spiral patterning algorithm, but the spiral direction was alternated each repetition (from groove center towards the walls and then from the walls towards the center). Such an algorithm gave bottom flatness equaling $10 \mu \mathrm{m}$ (rms st.dev.). The surface roughness of non-processed sample is $0.5 \mu \mathrm{m}$ (rms st.dev.). No saturation effects (decrease of ablation rate due to wall absorption and defocus) were observed at depths up to 400 $\mu \mathrm{m}$ with these pulse energies $(30 \mu \mathrm{J})$ and focusing conditions. It was found, however, that in order to get smooth grooves with steep slopes groove width to depth ratio should be at least 1:2 or less. With deeper cavities, the bottom roughness became slightly worse, mainly due to debris that becomes hard to remove even after several washings in ultrasonic bath.

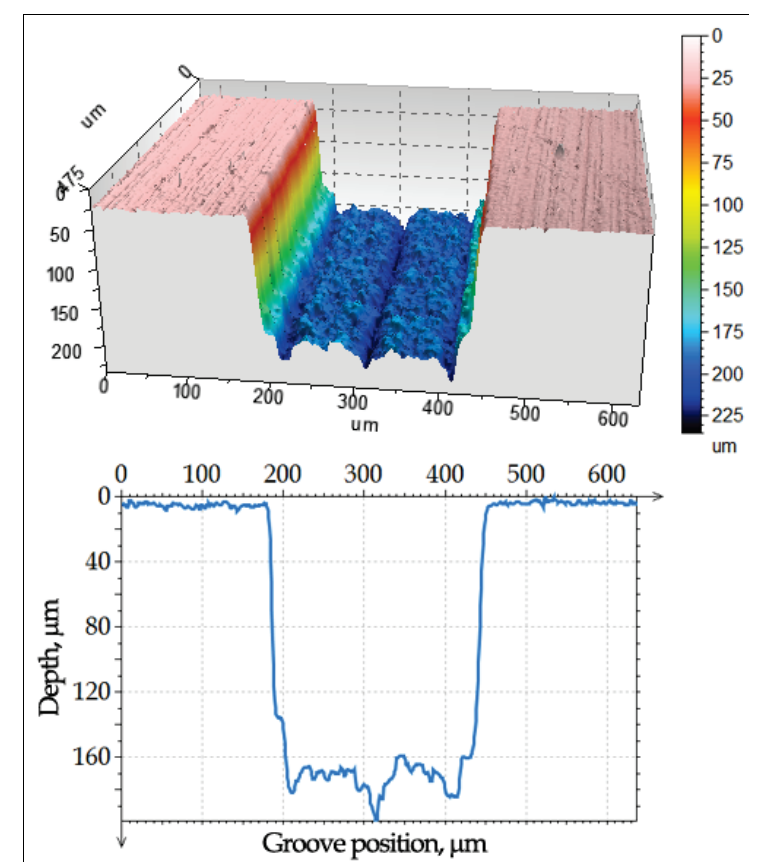

Fig. 4. Optimized flat-bottomed groove in steel made with spiral patterning algorithm. 


\subsubsection{Microfabrication using beam scanner}

In order to micromachine with galvanometric scanner, an f-theta lens with rather long focus length needed to be used. Here we used lens that had 100-mm focal length (nominal NA value of 0.02 ). The focused spot diameter in was approx. $40 \mu \mathrm{m}$. The larger spot size decreased laser fluency at focus and, respectively, ablation efficiency. We used $30-\mu \mathrm{J}$ laser pulses $(6 \mathrm{~W}$ at $200 \mathrm{kHz})$ in this experiment, which corresponds to $2-\mathrm{J} / \mathrm{cm}^{2}$ energy fluency at the focus. This value was in the gentle ablation regime [22].

Lower ablation efficiency requires greater number of patterning algorithm repetitions in order to achieve required depths. However galvanometric scanners do not have strict speed limitations, so processing speed could be much greater even taking in account higher repetition numbers. The scanning speed used here was $1000 \mathrm{~mm} / \mathrm{s}$. The typical groove cut in steel sample is shown in Fig. 5. We used spiral patterning algorithm (pitch size $5 \mu \mathrm{m}$ ) that was repeated 145 times. Overall manufacturing time for $1-\mathrm{cm}$ line groove was $120 \mathrm{~s}$. Thus comparing with linear stages, the processing time is higher almost by an order of magnitude. Manufactured grooves had steep walls; however, the bottom surface roughness was slightly worse and reached $20 \mu \mathrm{m}$ (rms, st.dev.). When increasing algorithm repetition number, groove tend to develop $\mathrm{V}$-shape form as shown in Fig. 6 (repetition number here was 500, and groove depth reached $900 \mu \mathrm{m})$.
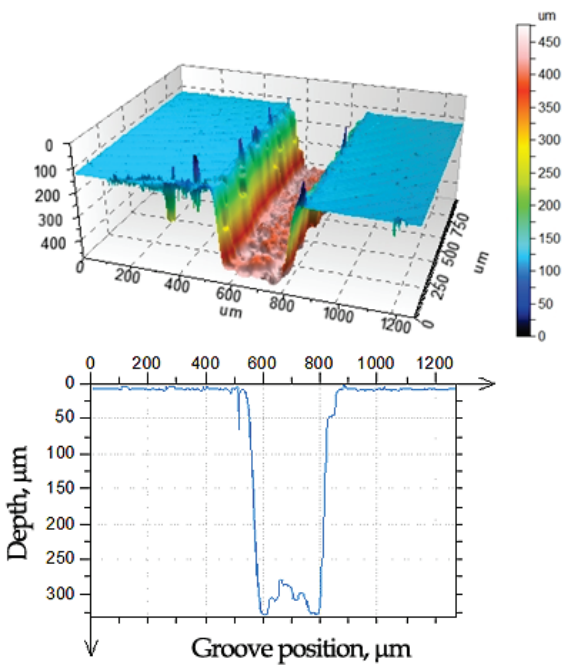

Fig. 5. Typical groove profile cut in steel sample using galvanometric scanners. Processing parameters: $6 \mathrm{~W}$ at $200 \mathrm{kHz}$. Scanning speed $1000 \mathrm{~mm} / \mathrm{s}$. Spiral patterning algorithm with 145 repetitions.

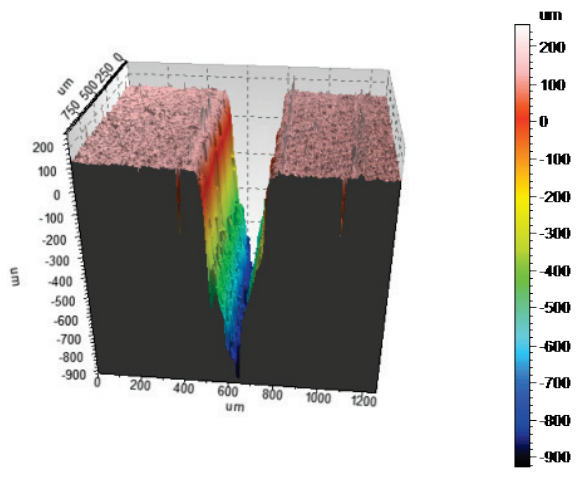

Fig. 6. Groove profile cut in steel with 500 repetitions.

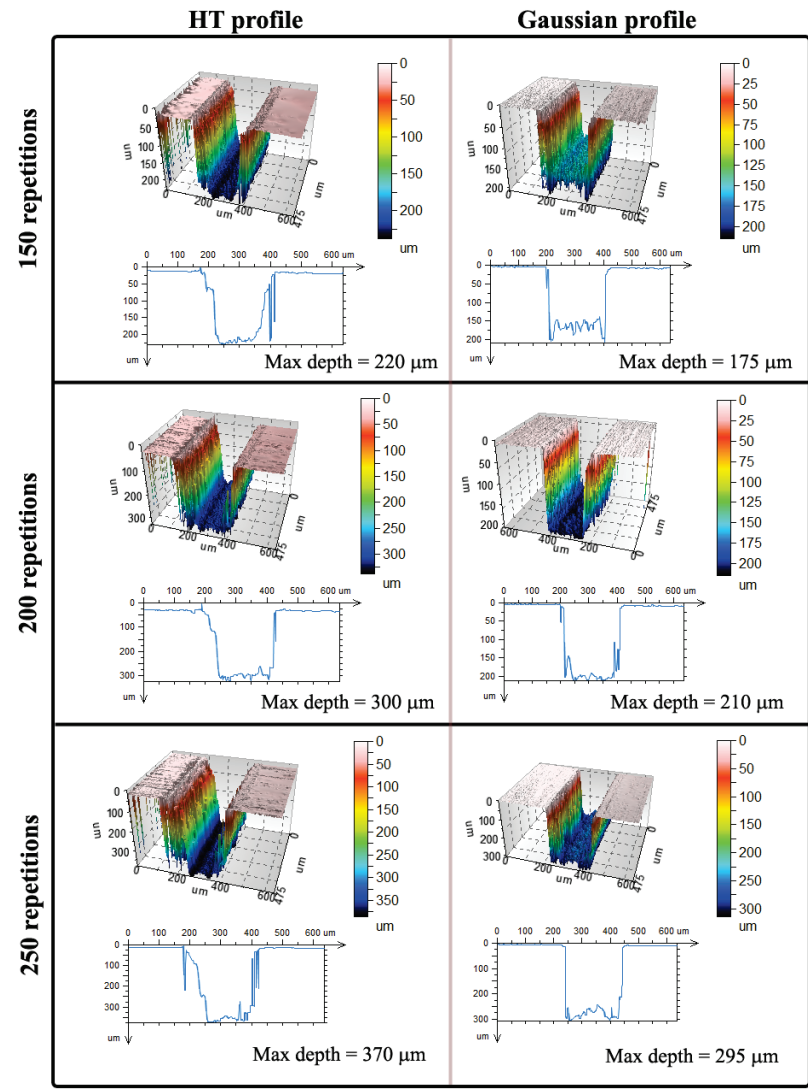

Fig. 7. The comparison of grooves cut with spiral algorithm (10 spirals with $5-\mu \mathrm{m}$ pitch) using Top-Hat (TH) and Gaussian beam profiles with different algorithm repetition numbers. Laser power - $5 \mathrm{~W}$, repetition rate $50 \mathrm{kHz}$, focused with $100 \mathrm{~mm}$ f-theta lens, scanning speed $1000 \mathrm{~mm} / \mathrm{s}$.

Also the comparison between grooves cut using Gaussian beam profile and Top-Hat $(\mathrm{TH})$ profile was carried out. Gaussian-to-TH beam conversion was realized using diffractive beam converter "FBS" (manufactured by TOPAG, $\mathrm{GmbH}$ ). The converter consists of the special hologram, recorded in the transparent material. When a Gaussian beam passes through this hologram, the central part of the beam (more energetic) is deflected close to the periphery in order to achieve homogeneous profile distribution. This beam shaper was inserted into the laser path in front of scanners. Detailed description of this beam shaping method can be found in a reference paper [23].

The comparison between groove profiles made with $\mathrm{TH}$ and Gaussian beams is shown in Fig.7. In this figure, 200$\mu \mathrm{m}$ width grooves manufactured in a steel sample using identical spiral writing algorithms are compared. It is clear that $\mathrm{TH}$ beam profile is capable of producing deeper grooves compared to the Gaussian beam, but groove quality is worse. Grooves made with Gaussian beam tend to have a flatter bottom and steeper, symmetrical walls. This indicates, that material ablation in the deep grooves is more accurately controlled by Gaussian beam than TH. Better ablated zone rectangularity with $\mathrm{TH}$ beam profile is achieved only when groove's depths are shallow (using only one repetition of patterning algorithm). 


\subsection{Laser Induced Breakdown Spectroscopy (LIBS) for the optimization of grooves micromachining}

Usually the result of a machining operation performed by ultrafast laser pulses is inspected manually after processing, which limits efficiency. In order to precisely control the machining process at the process level, monitoring of the workpiece under ablation is required. One promising technique is the use of laser-induced-breakdown spectroscopy (LIBS) [24, 25]. During laser-induced material breakdown, the intense femtosecond laser pulses ejects exited particles, which emit light. That emitted light can be collected and analyzed to identify the elemental composition of the material being ablated. Additionally, LIBS can be a reliable, quantitative technique for control of ultrafast laser micromachining processes.

We report the utilization of the LIBS for optimization of the groove micromachining by femtosecond laser pulses in steel. In our experiment, the collection angle, measured from the incident laser beam, is chosen to be the on-axis direction. The plasma emission which occurs during the laser ablation of the steel sample in air at ambient temperature and pressure was collected through a parabolic mirror and focused into an optical fiber. The fiber introduced the light into an imaging four channel spectrometer (AvaSpecUSB2-DT, Avantes) equipped with four diffraction gratings, 2400, 2400, 1800 and 1800 lines/mm providing spectral resolution of $0.09,0.07,0.1$ and $0.08 \mathrm{~nm}$ respectively. The spectra could be collected in a 200- to 664-nm spectral range. The spectrometer was equipped with a 2048-pixel CCD detector (Sony ILX554). The registered plasma emission spectra were analyzed using "Plasus SpecLine 2.1" software (Avantes).

During the micromaching process the LIBS signal intensity at some $\mathrm{Fe}$ I lines and $\mathrm{Cr}$ I line were monitored. It was observed that spectra at the beginning of the process, in the middle and at the end of it match one another well which shows that machining process and the material itself was quite uniform.

We measured LIBS signal intensity dependence on the number of the passes over the same groove (number of cuts) (Fig. 8) and on the grooves formation speed (Fig. 9). LIBS signal intensity is proportional to the volume of material being ablated.

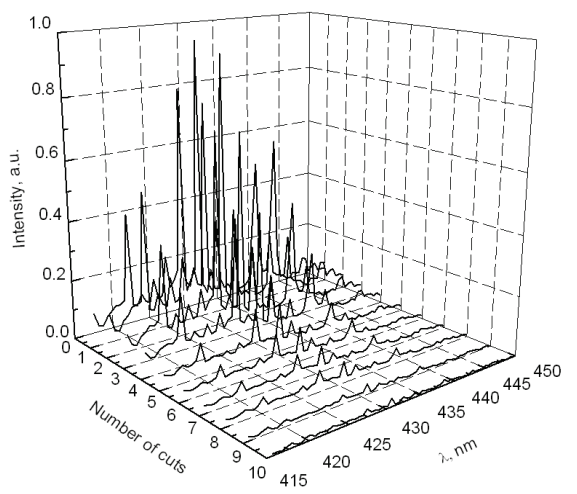

Fig. 8. Collected LIBS spectra of the steel sample in dependence on the number of the pass over the same groove.
At low groove formation speed a deeper groove is reached with the first two passes over the same groove. This is illustrated by the LIBS signal change in Figure 9a. The higher cutting speed the lower depth of the groove (less ablated volume of the material) is achieved per one pass over the groove. Therefore with higher cutting speed the LIBS signal changes less with an increasing number of cuts (Fig. 9).

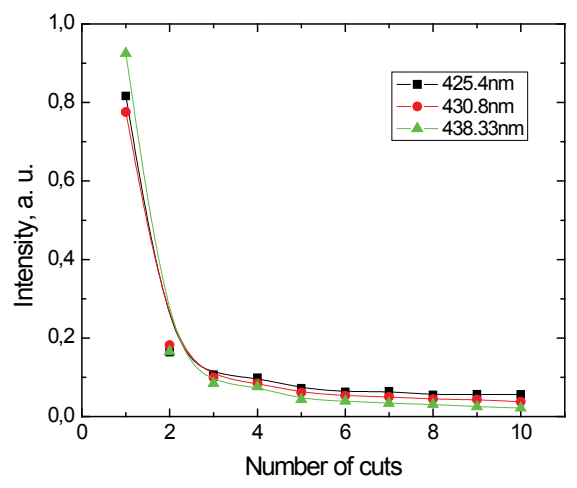

a)

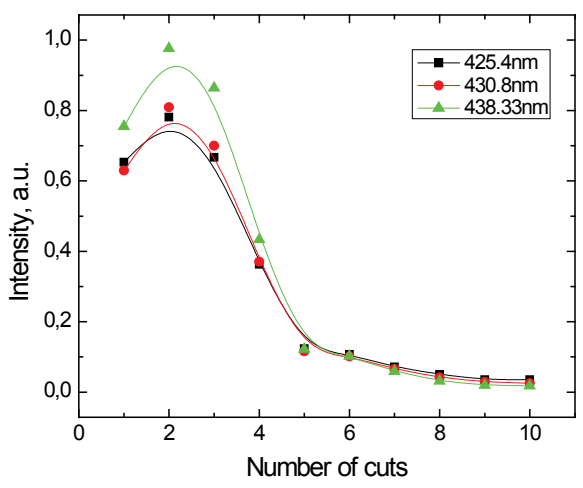

b)

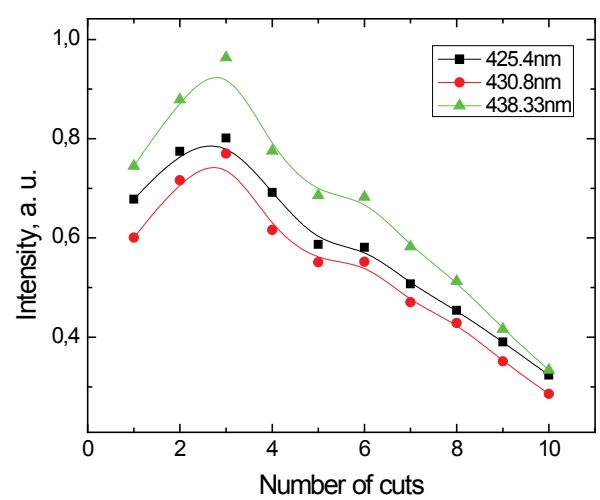

c)

Fig. 9. LIBS signal (selected Fe I lines (430.8 nm, 438.3 $\mathrm{nm})$ and $\mathrm{Cr}$ I line $(425.5 \mathrm{~nm})$ ) intensity dependence on the number of cuts at different grooves formation speed: a- $5 \mathrm{~mm} / \mathrm{s}$; b- $10 \mathrm{~mm} / \mathrm{s}$; c- $20 \mathrm{~mm} / \mathrm{s}$. 


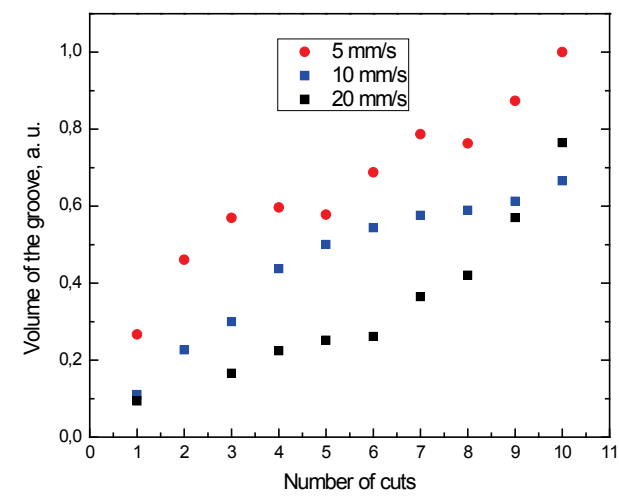

Fig. 10. The groove volume (which is proportional to the amount of the ablated mass of the material) dependence on the number of cuts at different grooves formation speed.

The depths of the grooves were measured with an optical profilometer (Sensofar PL $\mu$ 2300) after each pass over the same groove. The volumes of the grooves (which are proportional to the amount of the ablated mass of the material) were calculated. Figure 10 shows the calculated groove volume as a function of the number of passes for three different groove formation speeds. In the region of investigated, as the number of passes over the same groove increases, the volume increases nearly linearly. These values correlate with the LIBS signal. In this way the ablation efficiency could be monitored. Compared with passive machining processes without any feedback control, the LIBS signal intensity controlled micromachining approach provides several important advantages including less damage to the substrate layer, reduced machining time and more-uniform machining features.

\section{Conclusions}

In conclusions, we have demonstrated the formation of 30to $200-\mu \mathrm{m}$ width grooves with depth to width ratio up to 2 and steep slopes on a stainless steel surface by femtosecond laser ablation. Influence of the micromachining strategies, scanning methods, beam shapes on the precision and material removal speed was investigated. In order to monitor the micromachining process laser induced breakdown spectroscopy measurements were incorporated in the micromachining setup. It was concluded that optimized scanning pattern gives the best quality rectangular grooves with high precision in high fluency ablation conditions.

\section{Acknowledgements}

This work was partly supported by EC 7FP project MesMesh (contract number NMP3-SE-2009-229099). The authors acknowledge Prof. R. C. Eckardt for the editing. G. Chozevskis, J. Litvaityte acknowledge Student Research Fellowship Award from the Lithuanian Science Council.

\section{References}

[1] C. B. Schaffer, A. Brodeur, J. F. Garcia, E. Mazur, Optics Letters, 26, p. 93 (2001).

[2] M. Arrigoni, Photonics Spectra , 38, No 6, June, p.73 (2004).
[3] F. Dausinger, F. Lichtner, H. Lubatschowski (Eds): „Femtosecond Technology for Technical and Medical Applications, Topics in Applied Physics“ vol. 96, Springer, (2004).

[4] S. Bruneau, J. Hermann, M. Sentis, G. Dumitru, V. Romano, H. P. Weber, A. Semerok, W. Marine, Proc. SPIE, 5147, p. 199, (2003).

[5] B. N. Chichkov, F. Korte, J. Koch, S. Nolte, A. Ostendorf, Proc. SPIE, 4760, p. 19 (2002).

[6] S. Nolte, C. Momma, H. A. Jacobs, A. Tunnermann, B. N. Chichkov, B. Wellegehausen, H. Welling, J. Opt. Soc. Am. B, 14, 2716 (1997).

[7] R. Le Harzic, D. Breitling, M. Weikert, S. Sommer, C. Foehl, S. Valette, C. Donnet, E. Audouard, F. Dausinger, Appl. Surf. Sci. 249, 322 (2005).

[8] C. Y. Chien, M. C. Gupta, Appl. Phys. A 81, 1257 (2005).

[9] D. Bruneel, G. Matras, R. Le Harzic, N. Huot, K. König and E. Audouards, Optics and Lasers in Engineering, Volume 48, Issue 3, p. 268-271(2010).

[10] A. Ancona, F. Röser, K. Rademaker, J. Limpert, S. Nolte, and A. Tünnermann, Opt. Express 16, 8958 (2008).

[11] R. Le Harzic, H.Schuck, R.Buckle, T. Anhur, F.Bauerfeld, D.Sauer, Lasers in Manufacturing 2005, p. 485, Stuttgart: AT-Verlag (2005).

[12] G. Mourou, Appl. Phys. B. 65, 205 (1997).

[13] W. Krupke, IEEE J. Select. Topics Quantum Electron., 6, 1287 (2000).

[14] M. E. Fermann, M. Hofer, F. Haberl, and S. P. Craig-Ryan, Electron. Lett. 26, 1737 (1990).

[15] F. Roser, J. Rothhard, B. Ortac, A. Liem, O. Schmidt, T. Schreiber, J. Limpert, and A. Tunnermann, Opt. Lett. 30, 2754 (2005).

[16] S. V. Marchese, C. R. E. Baer, A. G. Engqvist, S. Hashimoto, D. J. H. Maas, M. Golling, T. Südmeyer, and U. Keller, Opt. Express. 16, 6397 (2008).

[17] E. Innerhofer, F. Brunner, S. V. Marchese, R. Paschotta, G. Arisholm, S. Kurimura, K. Kitamura, T. Usami, H. Ito, and U. Keller, J. Opt. Soc. Am. B 23, 265 (2006).

[18] B.N. Chichkov, C. Momma, S. Nolte, F. von Alvensleben, A. Tünnermann, Appl. Phys. A, 63, 109 (1996).

[19] F. Dausinger, Proc. SPIE, 4830, 471 (2003).

[20] J. König, S. Nolte, and A. Tünnermann, Opt. Express, 13, 10597 (2005).

[21] Ostendorf A., C. Kulik, T. Bauer, and N. Barsch, Proc. SPIE, 5340, 153 (2004).

[22] K. Kurselis, T. Kudrius, D. Paipulas, O. Balachninaite, and V. Sirutkaitis, Lithuanian Journal of Physics, Vol. 50, No. 1, p 95 (2010).

[23] G. Račiukaitis, E. Stankevičius, P. Gecys, M. Gedvilas, C. Bischoff, E. Jager, U. Umhofer, F. Volklein: J. Laser Micro. Nanoen., 6(1), 37 (2011).

[24] S. Klein, T. Stratoudaki, V. Zafiropulos, J. Hildenhagen, K. Dickmann, and T. Lehmkuhl, Appl. Phys. A 69, 441(1999).

[25] Tao Tong, Jinggao Li, and Jon P. Longtin, Appl. Optics, Vol. 43, No. 9, 1971 (2004).

(Received: June 30, 2012, Accepted: November 13, 2012) 